\title{
ON ARITHMETICAL CLASSIFICATIONS OF INACCESSIBLE CARDINALS AND THEIR APPLICATIONS
}

BY

\author{
GÉZA FODOR AND ATTILA MÁTÉ
}

\begin{abstract}
Lately several authors, among them Fodor, Gaifman, Hanf, Keisler, Lévy and Tarski, dug out an interesting and unduly forgotten operation of Mahlo that, loosely speaking, from a sequence of ordinals discards all those that are easy to locate in this sequence. The purpose of the se authors was to invent strengthenings and schemes for repetitions of this and similar operations and to study the properties of cardinals that can be discarded in this way when started with a specific class; for example, the class of all inaccessible cardinals. Our attempt here is to consider such schemes for repetitions of operations that can in a sense be described in an arithmetical way, which might also be called constructive; our investigations are akin to the problem of constructive description of possibly large segments of, say, the set of all countable ordinals. Some applications of our classifications scheme are exhibited, questions ranging from definability of inaccessible cardinals in terms of sets of lower ranks to incompactness theorems in infinitary languages. The paper is concluded with an algebraic-axiomatic type study of our scheme.
\end{abstract}

1. Introduction. In these notes we are going to define a set theoretical operation that selects from a given class of ordinal numbers, which is usually assumed to be the class of all inaccessible cardinals, classes of cardinals having interesting properties, such as the property of being nonmeasurable or strongly incompact, etc. (For the definitions of the concepts mentioned see the next section.) Several problems of this type were investigated earlier, as the references given at the end of this paper indicate.

The process we are going to define for generating cardinals with the enumerated properties is based on an earlier process defined by the first of the authors in [3], [4] and [5]; the process described in the present paper is actually to be considered as a generalization of Fodor's process, defined in an essentially simplified way. This simplication has, however, one disadvantage; namely, its price is that the intuitive motivation becomes almost completely lost. So to counteract this in $\S 3$, we describe in an informal way how the process was

Received by the editors October 23, 1969.

AMS (MOS) subject classifications (1969). Primary 0430, 0268; Secondary 0235, 0257.

Key words and phrases. a-complete ideal, a-truncation of the process, arithmetical classifications of cardinals, band, canonical process, canonical sequence, definability of inaccessible cardinals, divergent function, dropping•out, fixed-point operation, Fodor's process, hyper-inaccessible cardinal, Mahlo's process, measurable cardinal, nonmingling property, pseudo-universe, recollection step, regressive function, stationary-point operation, stationary set, strongly incompact cardinal, weakly compact cardinal. 
originally defined. Nevertheless, the reader may choose to skip this section completely if he desires.

The presentation of our process is intended to be made in a self-contained manner, i.e. for the understanding of the problems discussed here we try to dispense as much preliminary knowledge as possible; thus, for example, the reader is not assumed to be acquainted with the contents of the three papers of Fodor ment ioned earlier.

Operations on the class of all inaccessible cardinals were first considered by Mahlo in [10], [11] and [12] at the beginning of the 1900's. The relevance of his striking work to questions connected with the foundation of set theory was investigated only about half a century later. (See Lévy [9], Hanf [7], Keisler and Tarski [8], Gaifman [6] and the works of Fodor already cited.) The original procedure of Mahlo was expanded and generalized in three different ways in the cited works of Gaifman, Hanf and the first author of the present paper. While Gaifman's purpose was to generalize the operation used in one single step, the aim of the last two authors was to give a generalization for the frame in which the single operations are interated; the two different aims are easily combined.

We are going to make further comparison between Hanf's and Fodor's procedure. As to the former, one obtains a generalization of Mahlo's process by separating the two different roles played by the natural well-ordering of the ordinals in the original definition. Thus it is possible to define a process similar to, but more general than, that of Mahlo owing to the freedom we have in selecting one of the well-orderings. In this way we can make the process much more effective than it was originally, as long as we can find appropriate well-orderings of the ordinals.

Fodor's way of generalizing Mahlo's process was to repeat it a transfinite number of times; originally this was done in a very graphic manner by forming arrays of ordinals, such as transfinite-dimensional matrices. The reason why we abandoned this illuminating way of describing the process is that it renders more complicated the formal definition, and that the way actually chosen reveals some new aspects of the process as well as leaving the door open for its algebraic generalizations, such as that given in the last section.

Our comparison thus far shows that while Hanf gives a generalized scheme in which the original Mahlo process can also be described as a particular case, Fodor observes that there is a possibility of going much further than Mahlo did. Hanf's scheme seems to be more general in the sense that Fodor's process as actually defined in $\$ 5$ under the name "canonical process" can probably be described within its frame; this nevertheless does not make the latter superfluous. Indeed, in this content Fodor's process could also be viewed as the 
designation of particular well-orderings of the ordinals which implement Hanf's scheme particularly effectively. The snag in such a treatment of Fodor's process would partly be the loss of lucidity and intuitive motivation.

On the other hand, what was said immediately above about the relation between the generality of the two processes is only a guess, and before drawing a final conclusion it must be proved; and the hopes for proving such a result for the generalized forms of Fodor's process such as given in $\$ \$ 4$ and 10 are considerably less than in the special case. To boot, there are interesting theorems cut precisely on Fodor's process; an example for this is Theorem 8.2. The distinctive property of Fodor's process that makes this possible is that it can be uniquely characterized with the aid of a small number of axioms.

Such technical differences between the operations known for inaccessible cardinals as to whether they are presented in cumulative or discarding forms bear no significance. (Hanf's is an example of the first group, all the others mentioned of the second.)

As to the arrangement of this paper, it is divided into ten sections. The two preliminary ones are followed by an intuitive description of the process conforming with the way it was originally defined by the first author. This section may considerably help the reader in comprehending the ideas that led the authors to the concepts contained in further sections, but as remarked earlier, from a strictly mathematical viewpoint it may be entirely omitted.

In $\$ 4$ we outline some essential algebraic properties of the process, which is actually defined in the following section, where Lemma 5.1 might have some interest on its own account. In $\$ 6$ an operation called "dropping-out" is defined with the aid of the process, and $\$ 7$ revises the contents of the two preceding sections in a semiformalized style. $\$ \$ 8$ and 9 give special applications of the process, among them a result analogous to Hanf's about strongly incompact cardinals. $\$ 10$ given an algebraic generalization of the process, foreshadowed by the considerations of $\$ 7$. Here a generalization of the result, given in $\$ 9$, on strong incompactness of inaccessible cardinals is also obtained.

2. Preliminary concepts. In the present section we intend to give a complete account of the concepts used but not explained later. Of course we avoid repeating well-known notions of set theory and logic. As a matter of fact, we try to arrange the material here so that it could either be read through before the remaining sections, or entirely skipped and used only as a repository of references. So we present the material under identification numbers, which in the second alternative may prove useful. We hope to relieve our definitions with occasional poetic touches. 
2.1. The notations used below are the usual ones of logic and set theory. Set variables are denoted by lower case Latin letters, while those confined to ordinals are denoted by Greek ones. Further notational questions are made clear by the context.

2.2. Logics of sentences of infinite length. While talking about a logic we always mean a first order $\operatorname{logic}$, i.e. a logic where none of the relation symbols is quantified. The length of a logical formula is defined as the number of occurrences of the symbols present in it.

Apart from the ordinary finite-length formulas we shall also be concerned with formulas of infinite length, i.e. with formulas which possibly admit conjunction or disjunction of an infinite number of components, and where an infinite array of quantifiers of the same type (i.e. either purely universal or purely existential ones) is allowed. The number of variable symbols available is not limited.

As an abbreviation we shall use the concept of the language $L_{a}$, where $a$ is a cardinal number, simply for indicating the collection of all formulas of length $<a$. Satisfiability, truth-value and other semantical concepts for the logic $L_{\alpha}$ are easily extended. For further details see [7, p. 310], or [17].

The meaning of a logical formula containing free variables should, unless otherwise stated, be interpreted as if these were all universally quantified in front of it.

2.3. A structure is a well-ordered sequence of which the first element is a set of objects and the others are relation symbols defined on the object-set. In our discussions below we shall be concerned only with structures having a finite number of finitary relations.

2.4. Models for an axiomatic theory are structures satisfying the given axioms. In what follows our models may contain a specified binary relation $\epsilon$ endowed with certain interesting properties. In order to go into considerations concerning these properties, as sume that

$$
\langle A, \epsilon\rangle
$$

is a model for a certain theory, where the other possibly occuring relation symb̄ols are suppressed. The above model is said to be set-theoretical if for any two $x$, $y \in A$

$$
x \in y \rightarrow x \in y \text {. }
$$

If moreover for arbitrarily chosen $x$ and $y$ we have

$$
y \in A \& x \in y . \leftrightarrow \cdot x \in y,
$$

then our model is called natural. (Dots are used as in W. V. Quine, Mathematical logic, 1947 (rev. ed., Harper and Row, New York, 1962).)

In our discussions we shall be interested in those axiom systems which, 
if they are satisfiable, are satisfiable also by natural models. As known, a necessary and sufficient condition for this is that the axiom system in question should imply the axiom of extensionality

$$
(\forall x)(\forall y)(x=y \cdot \leftrightarrow \cdot(\forall z)(z \in x \leftrightarrow z \epsilon y)),
$$

as well as the axiom

$$
\sim\left(\exists x_{i}\right)_{i<\omega} \bigwedge_{i<\omega}\left(x_{i+1} \epsilon x_{i}\right) .
$$

This latter formula simply stipulates that the relation $\epsilon$ is well-founded, i.e. that there exists no infinite sequence decreasing with respect to it (see Mostowski [13, Theorem 3, p. 147], or, alternatively, Shepherdson [16, 1.5, p. 171].)

2.5. Ordinals are to be thought of as the sets of all smaller ordinals, according to the convention introduced by J. von Neumann in [14]. Cardinals are identified with their initial numbers.

2.6. The rank of a set is defined recursively as the least ordinal exceeding the ranks of all its elements. In particular, this definition implies that the only set of rank 0 is the empty one.

2.7. Inaccessible cardinals without any specifying attribute always means strongly inaccessible cardinals. Otherwise, this notion is used in two different senses. A cardinal is called weakly inaccessible if it is regular and has a limit index. A cardinal is called strongly inaccessible if no set-theoretical operation leads out of the set of all cardinals preceding it; or, listing explicitly the operations relevant in the definition, a cardinal $\alpha$ is strongly inaccessible if it is regular and for any cardinal $\beta<\alpha$ the inequality $2^{\beta}<\alpha$ holds.

Obviously an alternative way of defining strongly inaccessible cardinals is to say that a regular cardinal $\alpha$ is strongly inaccessible if and only if the set $R(\alpha)$ of all sets of rank $<\alpha$ along with the usual elementhood relation $\epsilon$ forms a natural model for the Zermelo-Fraenkel set theory.

2.8. Set theory is to be interpreted in one of the axiomatic theories. An upto-date account of these is presented e.g. in Cohen's book [1]. For our purposes we find it most natural to use the Zermelo-Fraenkel theory, although we use terms that cannot be interpreted in it directly. Thus, for example, while talking about classes we should imagine them as sets having a given large inaccessible cardinal $\alpha$ as rank while the ranks of the "real" sets are restricted to smaller ordinals. The cardinal $a$ here will often be referred to as the pseudo-universe.

An alternative but less convenient possibility is to use Bernays-type theories supplemented with some axioms providing for the existence of classes of classes ... of classes up to a certain finite degree.

When involved in discussions about large inaccessible cardinals we implicitly 
assume their existence, which could be formulated in terms of additional axioms, so-called strong infinity axioms.

2.9. An a-complete ideal, where $\alpha$ is a regular cardinal number, is a set of sets such that it contains any subset of its elements as well as any union of fewer than $\alpha$ of its elements. Calling the support of an ideal the union of all its elements, an ideal is said to be principal if it consists of all subsets of its support; it is called prime if of any two disjoint subsets of its support it contains at least one.

2.10. Measurable is a term applied to a cardinal $\alpha$ if it is the support of a nonprincipal a-complete prime ideal.

2.11. A cardinal $a$ is strongly incompact if there is a nonsatisfiable axiom system written in the language $L_{\alpha}$ containing $\alpha$ axioms such that any of its subsystems consisting of fewer than $\alpha$ axioms is satisfiable.

As is well known, every measurable cardinal is weakly compact, i.e. not strongly incompact (see Tarski [18]). The strongest result known asserting the strong incompactness of certain inaccessible cardinals is due to Hanf (see [7, Theorem 5, p. 319]).

2.12. A band is a set of ordinals such that it is closed under the natural ordering topology except that it need not contain its supremum.

2.13. Stationary (with respect to the ordinal a not cofinal to any ordinal $\leq \boldsymbol{N}_{0}$ ) is applied to a set of ordinals preceding $\alpha$ if it intersects all the sets that are bands with supremum $\alpha$. An alternative definition for stationary sets will be given below, where some of their essential properties will also be listed.

2.14. A regressive function is a function $f$ defined on some set of ordinal numbers such that for any ordinal number $a$ different from zero in its domain we have $f(\alpha)<\alpha$.

2.15. Divergent ( $w$ ith respect to the ordinal $\alpha$ ) is applied to a function $f$ defined on a set of ordinals less than $\alpha$ if

$$
(\forall \eta)(\exists \lambda)(\forall \xi)(\xi>\lambda \rightarrow f(\xi)>\eta),
$$

where Greek letters denote variables ranging over ordinals preceding $\alpha$ and, moreover, $\xi$ is confined to the domain of $f$. If $\alpha$ is a regular cardinal number, then this condition simply means that $f$ takes no value a times.

2.16. Stationary (with respect to the ordinal $a$ ) (1) is applied to a set of ordinals $<\alpha$ (this is an alternate definition) if it is not the domain of any regressive function that is divergent with respect to $\alpha$. The equivalence of the two definitions is confirmed by a theorem of Neumer (see [15, Satz 2, p. 257]).

(1) Whereas this definition can also be applied for ordinals a confinal to an ordinal $\leq \boldsymbol{x}_{0}$, it implies that there exists no stationary set with respect to these. 
From this second definition it is obvious that

Theorem 2.16. 1. The sets nonstationary with respect to a regular cardinal a $>\boldsymbol{\kappa}_{0}$ form an a-complete ideal.

The first author of this paper sharpened this result as follows (see [2, Hilfsatz, p. 140]):

Theorem 2.16. 2. Suppose $a$ is a regular cardinal $>\kappa_{0}$. Then, with respect to $\alpha$, the union of a mutually disjoint nonstationary sets is nonstationary if and only if the set formed by their initial elements is not stationary either.

This theorem can be equivalently stated in a form that generalizes the mentioned theorem of Neumer (see [2, Satz 2, p. 141]).

Theorem 2.16. 3. Suppose $\alpha$ is an uncountable regular cardinal. Then any regressive function defined on a set stationary with respect to $\alpha$ is constant on a subset stationary with respect to a of its domain.

2.17. The fixed-point operation performed on a set $H$ of ordinals results by definition in the set $\mathrm{fp}(H)$ of all ordinals that both belong to $H$ and are limit points of it in the natural ordering topology.

2.18. The stationary-point operation performed on a set $H$ of ordinals results in the set sp $(H)$, containing exactly those elements $\alpha$ of $H$ for which the set $H \cap \alpha$ is stationary with respect to $\alpha$.

3. An informal description of the original form of the process. In order to compensate the loss of intuitive motivation suffered in the syntactic simplification of the definition of the process, we give now an informal account of how it was originally defined by Fodor in [3], [4] and [5]. We in no way aim at completeness here, since the concepts presented now will be defined precisely later in a more general form.

The process depends on an operation $f$ with classes of ordinals as range, and actually is a scheme for repeating it transfinitely many times. To be more specific, choose $f$ as the fixed-point operation defined at the end of the previous section, and start with the class of all inaccessible cardinals, ordered by magnitude and considered as a row or as a one-dimensional matrix of ordinals. Additional rows can be obtained in turn by applying the fixed-point operation repeatedly (see 2.17 in the previous section). We can continue this repetition even transfinitely if in a step corresponding to a limit ordinal we simply take the settheoretical intersection of all rows obtained previously. If we run through all the ordinals, then we obtain a well-ordered sequence of rows all but the first member of which we build into a two-dimensional matrix. 
Now take the fixed points of the first column of the first matrix obtained as the first row of the second matrix and apply to it the same procedure. Using the fixed points of the first column of this matrix, a third matrix can be produced. This operation can be repeated transfinitely many times if in a step corresponding to a limit ordinal the first row of the matrix to be defined is the intersection of the first rows of all preceding matrices. By running through all the ordinals we obtain a sequence of two-dimensional matrices, which, with the exception of its first member, we build into a three-dimensional matrix.

Taking the fixed points of the class of the first elements of the two-dimensional matrices in this three-dimensional one we obtain a row with which we can restart again to build another three-dimensional matrix, etc. transfinitely. There is no problem in continuing this process in order to get a second three-dimensional matrix, etc., or indeed in continuing it while only finite-dimensional matrices enter. When all finite-dimensional matrices have been constructed, we can take the intersection of the classes of ordinals contained in the first $n$-dimensional matrices $(n=2$, $3, \ldots)$, and restart the whole process with the fixed points of this class. Thus we obtain another lot of finite-dimensional matrices as above, which we build into one infinite-dimensional matrix.

Proceeding this way we obtain a sequence of matrices whose dimensionalities are the ordinals in turn. Now there is a possibility of restarting the procedure with the fixed points of the class of the first elements of the first matrix of each dimensionality, etc. Having performed the above process up to a certain stage we are interested in the ordinals that have not dropped out by then. This simply means the ordinals that either occur in the lastly obtained matrix as elements or have "chances" of reentering later by the recollection part of the process. Such ordinals are inaccessible in a very strong sense.

As also mentioned in $\$ 2$, we do not have to perform the above process with proper classes of ordinals, but can choose a large inaccessible cardinal as pseudouniverse and consider only smaller ordinals instead of all of them. An obvious but important feature of the process described is that its effect on ordinals less than the chosen pseudo-universe is independent of the particular choice of this latter.

The device by which we can redefine the process consists in combining the transfinite "subdefinitions" of the various matrices in a single transfinite induction. To be able to achieve this it is necessary to define a well-ordering by which the transfinite induction proceeds. This is actually more complicated than the order type of all ordinals (or of all those preceding the pseudo-universe). In view of the fact that the ordering in question has to be defined constructively in terms of ordinals, the definition of the process is connected with the problem of 
constructive definition of ordinals; this aspect of the process will not, however, be touched upon in this essay.

4. A family of processes. In order to redefine our process with the aid of one single transfinite induction we shall linearize it, i.e. we shall rearrange the classes (obtained in the original definition as rows of matrices) in a linear wellordering. This, for the convenience of the reader as well as in order to obtain a deeper insight, will be accomplished in two steps: In the first step we give a definition that does not determine our process up to an isomorphism, i.e. which yields a family of processes; in the second step accomplished in the next section we specify a member of this family as the canonical process.

To obtain an arbitrary member of the mentioned family we proceed as follows: We choose a large inaccessible cardinal $V$ for playing the role of the pseudouniverse and consider an ordinal $S \leq V^{+}$, where $V^{+}$denotes the immediate succe ssor cardinal of $V$. To each element $\sigma$ of $S$ cofinal to $V$ we assign an increasing sequence $s(\sigma)$ of type $V$ of ordinals tending to it, and we require the fulfillment of the following condition, expressed vaguely by saying that no sequences mingle.

Of two sequences assigned to ordinals, either one is situated between two adjacent elements of the other or all elements of one precede every element of the other. (Cf. The orem 4.1 below.)

The process will be a scheme, determined by the assigned sequences, for the repetition of an operation $f$ which makes the correspondence to each set of ordinals one of its subsets. It seems reasonable to consider only monotonic operations which are stronger than the fixed-point operation, i.e. for which

$$
x \subseteq y \cdot \rightarrow \cdot f(x) \subseteq f(y)
$$

and

$$
f(x) \subseteq \mathrm{fp}(x)
$$

for any sets $x$ and $y$ of ordinals.

Starting with a set $H$ of ordinals $<V$, and denoting by $F(\sigma, H)$ the set obtained at the step $\sigma$ of the process, for an ordinal $\sigma$ preceding $S$, we define the process as follows:

For a start we put

$$
F(0, H)=H,
$$

and if $\sigma \neq 0$ is an ordinal $<S$ not cofinal to $V$, then we write:

$$
F(\sigma, H)=\bigcap_{\lambda<\sigma} \bigcup_{\lambda \leq \xi<\sigma} f(F(\xi, H)),
$$


i.e. we take the superior limit of the sets written on the right-hand side as $\xi$ runs over all the ordinals $<\sigma$. Finally, for $\sigma<S$ cofinal to $V$ we define the set obtained at the step $\sigma$ as the result if we perform the operation $f$ on the set of the initial elements of the sets obtained at the steps corresponding to the elements of $s(\sigma)$, i.e., $\min x$ denoting the initial element of the set $x$ of ordinals, we put

$$
F(\sigma, H)=f(\{\min F(\xi, H): \xi \in s(\sigma)\}) .
$$

As is easy to see from the above definition, for a successor ordinal $\sigma+1<$ $S$ we have

$$
F(\sigma+1, H)=f(F(\sigma, H)) .
$$

Now we prove two theorems.

Theorem 4.1. The assignment of the sequences $s(\sigma)$ to all ordinals $\sigma<S$ cofinal to $V$ can be carried out in the desired way if and only if $S<V^{+}$.

Proof. The function which makes the correspondence to each ordinal $\sigma<S$ cofinal to $V$ the second element of the assigned sequence $s(\sigma)$ is one-to-one and obviously regressive; these also imply that it is divergent with respect to $V^{+}$; therefore its domain is not stationary with respect to $V^{+}$. This excludes the case $S=V^{+}$.(2)

Conversely, in case $S<V^{+}$we can rearrange the set of all ordinals $<S$ cofinal to $V$ so as to have order type $\leq V$; hence an assignment of the desired kind is easily constructed successively by transfinite induction. In fact, assume that for all the ordinals $\xi$ preceding $\sigma$ in the considered rearrangement the sequences $s(\xi)$ have already been constructed. Then it is simply verified that $\sigma$ is not a limit point of the set

$$
\bigcup_{\xi<\sigma} s(\xi)
$$

Thus if we take any increasing sequence of ordinals tending to $\sigma$, an appropriate upper section of it can be chosen as $s(\sigma)$.

Theorem 4.2. Choose as the operation $f$ in the process defined above the fixed-point operation $\mathrm{fp}$. Then for any ordinal $\sigma<S$ the set $F(\sigma, H)$ differs from $H$ only in a set nonstationary with respect to $V$. Thus, in particular, if $V$ is a byper-inaccessible cardinal of type one and $H$ is the set of all inaccessible cardinals preceding $V$, then our process never terminates in the sense that aforementioned sets will be empty.

(2) If $\alpha<\beta$ are regular cardinals, then the set of all ordinals $<\beta$ cofinal to $\alpha$ is stationary with respect to $\beta$. Indeed, the ath element of any set which is a band with respect to $\beta$ belongs to this set. 
An inaccessible cardinal is hyper-inaccessible of type one, according to Lévy's definition given in [9], if the set of all inaccessible cardinals preceding it is stationary with respect to it. Thereby the second part of the above theorem is easily established as a consequence of its first part. Thus we need turn our attention only to the proof of the first assertion.

Proof of the theorem. The statement is easily proved by transfinite induction -we only have to check the various types of steps in the definition of the process.

To start with, at a step corresponding to a successor ordinal $\sigma+1<S$ the set

$$
F(\sigma, H)-F(\sigma+1, H)=F(\sigma, H)-\mathrm{fp}(F(\sigma, H))
$$

is nonstationary in view of the definition of the fixed-point operation; therefore the set

$$
H-F(\sigma+1, H)
$$

is not stationary, by induction. Similar conclusions can be drawn for steps corresponding to a limit ordinal $\sigma<S$ with the aid of Theorem 2.16.2 or 2.16.1 according to whether $\sigma$ is or is not cofinal to $V$. This completes the proof.

5. The canonical process. In order to describe univocally a member of the family of processes defined in the previous section it is necessary to specify assignment of the sequences $s(\sigma)$, where $\sigma$ is an ordinal cofinal to $V$ and smaller than $S$. Here the ordinal $S<V^{+}$is selected large, if possible, but in doing so we are obviously restricted by the increasing difficulties arising in the definition of the sequences $s(\sigma)$ as $\sigma$ becomes large. Among the processes so obtained there is one which can equally be derived by the matrix method described in $\$ 4$; in the sequel this will be called the canonical process. In the concluding section there will be some discussion on what makes this particular process preeminent among the others.

In the definition given below $S$ will be, not an ordinal, but only a wellordered set. This simplifies our considerations essentially and, on the other hand, has no theoretical significance. The order type of $S$, which roughly determines how far we continue the process, will exceed considerably the pseudouniverse $V$. We do not aim at exploiting fully possibilities in this direction at the first go, since this would entail abandoning much of the simplicity here. At the end of this section we shall show how to extend this first stage of the canonical process considerably, though further canonical extensions might be possible. To check this assertion, and mainly, if this is the case, to decide it in a negative direction is rather difficult as long as we do not give a mathematical formulation of the problem by defining precisely the yet intuitive notion of the 
canonical process. Only partial results are obtained in this latter question in the last section.

Before we actually set about defining the set $S$ we prove a lemma, which will help us in establishing its well-orderedness, but which we think worthwhile discussing independently of our specified course because of its own interest. Let $P$, therefore, be an arbitrary well-ordered set, and let $S$ be the set of all its finite subsets. Define an ordering in $S$ as follows: Of two elements of $S$ we consider as greater whichever contains the maximal element in the ordering of $P$ from their symmetrical difference. We then have

Lemma 5.1. The ordering of $S$ as defined above is a well-ordering.

Proof. (3) The minimal element of an arbitrary subset $A$ of $S$ can be found as follows. Taking $A_{1}=A$, and assuming that the sets $A_{j}$ have already been defined for all positive integers $j \leq i$, define $A_{i+1}$ in two steps: first single out the smallest element of $P$ which is maximal in some element of $A_{i}$. Then consider those elements of $S$ which contain this singled-out element as maximal; omit from each of them this latter element and take the set of obtained sets as $A_{i+1}$. This procedure must terminate in a finite number of steps by obtaining an $A_{i}$ containing 0 . Indeed, the singled-out elements form a descending sequence of a well-ordered set. The set of all these will constitute the minimal element of the starting set $A$.

Returning to our specific problem, the set $P$ will be defined as the set of all pairs

$$
\left(\xi_{0}, \xi_{1}\right)
$$

of ordinals $<V$, well-ordered in a reverse lexicographical manner such that

$$
\left(\vartheta_{0}, \vartheta_{1}\right)<\left(\xi_{0}, \xi_{1}\right)
$$

holds if and only if either $\boldsymbol{\vartheta}_{1}<\xi_{1}$ or $\vartheta_{1}=\xi_{1}$ and $\vartheta_{0}<\vartheta_{1}$.

As a deviation from the general considerations before, the set $S$ will here be only a proper subset of what it was before. Specifically, $S$ is defined as the set of every finite subset of $P$ which does not simultaneously contain two pairs differing only in the first ordinal constituting them. The definition of the ordering of $S$ will be derived from that of $P$ in the same way as before, and on account of the lemma just proved we see that $S$ is actually well-ordered thereby. For this well-ordering we adopt the symbol $\prec$.

What now remains in order to accomplish the definition of the canonical process is to assign the sequence $s(x)$ to each element $x$ of $S$ cofinal to $V$,

(3) This short proof is due to A. G. Hughes, University of Szeged, Hungary. 
meaning by this phrase the elements $x$ of $S$ for which the order type of the set

$$
\{y \in S: y \prec x\}
$$

is cofinal to $V$. To achieve this we write the element $x$ in the form

$$
\{p\} \cup y,
$$

where the pair $p$, the smallest element of $x$ in the ordering of $P$, is detached.

It is easy to verify that $x$ is cofinal to $V$ if and only if the first ordinal constituting $p$ is a nonlimit one (i.e. either zero or a successor ordinal), and the second is a successor ordinal; that is, if $p$ has one of the following forms:

$$
\left(0, \lambda_{1}+1\right)
$$

or

$$
\left(\lambda_{0}+1, \lambda_{1}+1\right) .
$$

The assigned sequences $s(x)$ are then defined, accordingly, as

$$
\left\{\left(a, \lambda_{1}\right)\right\} \cup y
$$

or as

$$
\left\{\left(\alpha, \lambda_{1}\right)\right\} \cup\left\{\left(\lambda_{0}, \lambda_{1}+1\right)\right\} \cup y
$$

where $a$ runs over all ordinals $<V$.

Thus, having defined the assigned sequences, the definition of the canonical process is completed. Nevertheless, it may be interesting to point out that the set

$$
F(x, H)
$$

obtained at a step $x$ of the process can be defined recursively by a single formula. Without going into deeper details we mention that if, detaching its minimal element, $x$ has the form

$$
\left\{\left(\xi_{0}, \xi_{1}\right)\right\} \cup y
$$

then, suppressing the starting set in the notation of the sets obtained at the steps of the process by writing $F(\cdot)$ instead of $F(\cdot, H)$, we can easily derive ( $\min K$ for a set $K$ of ordinals denotes the initial element of this set, as before):

$$
F(x)=f(F(y)) \cap \bigcap_{\vartheta_{0}<\xi_{0}} f\left(F\left(\left\{\left(\vartheta_{0}, \xi_{1}\right)\right\} \cup y\right)\right)
$$

$$
\begin{aligned}
& \cap \bigcap_{\vartheta_{1}<\xi_{1}} f\left(\left\{\min F\left(\left\{\left(\alpha, \vartheta_{1}\right)\right\} \cup y\right): a<V\right\}\right) \\
& \cap \bigcap_{\vartheta_{0}<\xi_{0}} \bigcap_{\vartheta_{1}<\xi_{1}} f\left(\left\{\min F\left(\left\{\left(\alpha, \vartheta_{1}\right)\right\} \cup\left\{\left(\vartheta_{0}, \xi_{1}\right)\right\} \cup y\right): a<V\right\}\right) .
\end{aligned}
$$


Here the intersection of no sets should be disregarded or interpreted as the whole universe of sets.

As a matter of fact, the complicatedness of the above formula is some what illusory. More exactly, depending on the pair

$$
\left(\xi_{0}, \xi_{1}\right) \text {, }
$$

most parts of it can be disregarded. In fact, only the first, second, third or fourth component of the intersection on the right-hand side of this formula needs to be considered according as we have $\xi_{0}=0, \xi_{1}=0 ; \xi_{0} \neq 0, \xi_{1}=0 ; \xi_{0}=0, \xi_{1} \neq 0$; or $\xi_{0} \neq 0, \xi_{1} \neq 0$; respectively.

As a concluding act in this section we remark that the canonical process can indeed be continued much further than it is defined above. The way this can be done is the following: we define the set $S^{*}$ of all steps of the enlarged canonical process as the set of all sequences

$$
\left(x_{0}, x_{1}, x_{2}, \ldots\right)
$$

of length $\omega$ of elements of $S$ such that only a finite number of the $x$ 's differs from the empty set, which is the initial element of $S$. The well-ordering of $S^{*}$ will be defined in the reverse lexicographical way as follows: Of two elements of $S^{*}$,

$$
\left(x_{0}, x_{1}, x_{2}, \ldots\right) \text { and }\left(y_{0}, y_{1}, y_{2}, \ldots\right)
$$

we say that the first precedes the second if for some integer $i$ we have $x_{i} \prec$ $y_{i}$ and $x_{j}=y_{j}$ for any larger $j$.

We still have to define the assigned sequences in $S^{*}$. This can be done in the following manner: If $i$ is the smallest integer such that $x_{i} \neq 0$ in the element under (5.13) of $S^{*}$ then we distinguish three cases:

A. If there is a sequence assigned to $x_{i}$ as an element of $S$, then denoting the $a$ th element $(\alpha<V)$ of this sequence by $y_{\alpha}$, we assign to the considered element the sequence of the elements

$$
\left(0, \ldots, 0, y_{\alpha}, x_{i+1}, \ldots\right) \quad(\alpha<v) .
$$

B. If $i>0$ and the element $x_{i}$ is the immediate successor of $y$ in the ordering of $S$, then the elements of the assigned sequence will be in turn

$$
\left(0, \ldots, 0,\{(0, \alpha)\}, y, x_{i+1}, \ldots\right) \quad(\alpha<V) .
$$

C. Finally, if neither of the above cases occurs, then the element in question is not cofinal to $V$ in the ordering of $S^{*}$, and therefore the assigned sequence will not be defined.

As we have already mentioned at the beginning of this section, it might well 
turn out that the above enlarged version of the canonical process still does not go as far as possible in principle, but this question will not be investigated in the present paper.

6. The operation derived. As already mentioned in the introduction, with the aid of our process we can define an operation which when performed on a class of inaccessible cardinals results in a scarcer set of inaccessible cardinals. As yet, however, only a vague reference was given in $\$ 3$ as to how we intend to define this operation: namely, we mentioned there that the result of the operation will be the class of all ordinals which do not drop out in the course of the process. We are now going to define precisely what dropping-out means for the canonical process.

As foreshadowed at the cited place in $\S 3$, we say that an ordinal drops out in the course of the canonical process if it cannot occur in the sets $F(x, H)$ after a certain step $y$; the phrase "cannot" used instead of, for example, "does not" intends to express that the ordinal spoken of would not reenter the process even if we were to continue it in a consistent way. This, however, should not be considered as a definition. It is only intended to be an intuitive justification of the definition given below. There, as well as in the rest of the whole paper, the phrase "canonical process" can mean the processes defined in the previous section having either $S$ or $S^{*}$ as the set of steps. Whereas the second of the alternatives delivers generally stronger results, it is much simpler to think in terms of the first one.

Definition 6.1. The ordinal $\xi \in H$ is said to drop out in the course of the canonical process if there exists a step $x$ of rank $<\xi$ such that

$$
\xi \notin F(x, H) \text {. }
$$

The first such step $x$ is called the dropping-out place of $\xi$, and $\xi$ is said to drop out at the step $x$.

In the above definition the condition rank $x<\xi$ could be substituted by one saying the ordinals constituting $x$, as described in $\$ 5$, all precede $\xi$. This might entail an inessential shift of the dropping-out places. On the other hand, it is easily seen that the definition given satisfies the requirement that an ordinal once dropped out does not reenter the process later. In fact, if such an ordinal is recollected after its dropping-out place, it is discarded in the same step by the operation $f$ provided previous assumptions such as that the starting set $H$ consist of inaccessible cardinals and that the operation $f$ be stronger than the fixed-point operation are satisfied.

As an easy consequence of the above definition we have the following 
Theorem 6.2. Choose as the operation $f$ the fixed-point operation fp. Then the set of all ordinals that drop out in the course of the canonical process is nonstationary with respect to the pseudo-universe $V$.

The assertion of this theorem is a strengthening of the result obtained in Theorem 4.2 in the particular case of the canonical process. Along with its corollary it can be considerably generalized, as pointed out in the last section.

Proof of the theorem. Assume that the ordinal $\xi$ is the ath ordinal in order of magnitude that drops out at the step $x$, and define $b(\xi)$ as the greater of $\alpha$ and rank $x$. It is easily seen that $b$ is then a regressive function, divergent with respect to $V$, defined on the set of all ordinals dropping-out. This proves the assertion.

As a corollary of the above theorem we obtain

Corollary 6.3. The operation derived from the fixed-point operation by the canonical process is strictly weaker than the stationary-point operation.

The phrase above that one operation is strictly weaker than the other refers to the fact that whenever they are performed on the same set the resulting set obtained by the weaker operation is strictly included in the one obtained by the other, unless both of them are empty.

The proof of the corollary is based upon the following lemma.

Lemma 6.4. If $H$ is a set stationary with respect to an ordinal $V$, then the set of all nonstationary points of $H$, i.e. the set

$$
H-\operatorname{sp}(H)
$$

is also stationary with respect to $V$.

This lemma, in the special case where $H$ is the set of all inaccessible cardinals preceding a hyper-inaccessible cardinal of type at least one, was communicated to the authors in a letter by R. Solovay.

Proof. Suppose, on the contrary, that there exists a set $X$ which is a band with respect to $V$ and does not contain any nonstationary point of the set $H$. Then the set of all fixed points of $X$ is also a band with respect to $V$, because $V$ is not cofinal to any countable ordinal (cf. footnote (1) to 2.16). Since $H$ is stationary with respect to $V$, the band $\mathrm{fp}(X)$ must intersect it, and, according to the above assumption, any point of the set

$$
\mathrm{fp}(X) \cap H
$$

must belong to $\mathrm{sp}(H)$. Assume that $\alpha$ is the initial element of this intersection. Then, as $\operatorname{cf}(\alpha)>\omega$, 


$$
\mathrm{fp}(X) \cap \alpha
$$

is a band with respect to $\alpha$ and it does not meet the set $H \cap \alpha$; this contradicts the fact that $\alpha$ is a stationary point of $H$.

Proof of Corollary 6.3. It is rather obvious that, starting with an arbitrary set $H$, the canonical process repeating the fixed-point operation, performed on this set, cannot discard any stationary point of $H$. In fact, this immediately follows from Theorem 6.2 if we choose the pseudo-universe $V$ as the first assumedly discarded stationary point of $H$. (As is easily seen, in the given particular case the effect of the process on an ordinal does not depend on the pseudo-universe as long as this given ordinal precedes the latter.) So we see that the operation derived from the fixed-point operation with the aid of the canonical process is not stronger than the stationary-point operation. That it is strictly weaker than this latter is shown by Lemma 6.4 compared to Theorem 6.2. In fact, the se show that it cannot discard all nonstationary points of $H$.

7. Formalization of the canonical process. As was already pointed out in the preceding section, in case we repeat the fixed-point operation the notion of dropping-out, and, indeed, the course of the whole canonical process, do not depend on how large the pseudo-universe is, as long as it exceeds the considered elements of the starting set $H$. If, however, we choose other operations to be repeated, the situation may radically change. In fact, the spoiling factor is that the behaviour of the operation $f$ in the definition of the process might be changed if we apply it to different lower sections of a given set. In order to circumvent this trouble we explicitly exclude the above possibility by requiring that the operation $f$ satisfy

$$
(\forall x)(\forall \xi)(\xi \in f(x) \leftrightarrow \xi \in f(x \cap \xi+1)) .
$$

Thus the functions considered here, i.e. those satisfying the above property together with (4.1) and (4.2) essentially coincide with the monotone local thinning functions described in the cited paper of Gaifman. It is important to note that the specific functions considered, namely the fixed-point and stationarypoint operations, satisfy all the properties in question.

Confining ourselves henceforth only to the class of functions described in the previous paragraph, the course of the canonical process becomes independent of the pseudo-universe; therefore the presence of the latter in its definition is unaesthetical. To get around this situation, there is a possibility of eliminating the pseudo-universe with the aid of formalization. The formalization of the process, which can be entirely carried out in the frame of ZermeloFraenkel set theory, is also necessary for the applications given in the next two sections, as well as for its generalization given in $\$ 10$. 
This formal recasting of the definitions given in the previous section will be based on the ternary predicate $\Sigma, \Sigma x t u$ expressing that $x$ and $u$ are elements of the set $S$ defined in $\$ 5, t$ is an ordinal, and moreover $u$ is the th element of the sequence $s(x)$, assigned to $x$ in the way defined before. (4) We shall, however, somewhat extend this definition. Precisely, the truth value of $\Sigma x t u$ will be defined as follows. We stipulate it to be false unless $t$ is an ordinal and, using the terminology and the notations of $\$ 5, x$ and $u$ are of the same form as the elements of the set $S$, except that the ordinals constituting them are not limited from above, i.e. are not required to be smaller than a certain pseudo-universe. In this latter case we detach the smallest pair in $x$ as done under (5.4), and so we write

$$
x=\left\{\left(\xi_{0}, \xi_{1}\right)\right\} \cup y .
$$

Now we distinguish several cases when the truth value of $\Sigma x t u$ will be defined as "true". First we consider those in which the sequence $s(x)$ was defined in the earlier discussion.

A. $\xi_{0}=0$ and $\xi_{1}$ is a successor ordinal, i.e. $\xi_{1}=\lambda_{1}+1$ for some $\lambda_{1}$, and

$$
u=\left\{\left(t, \lambda_{1}\right)\right\} \cup y \text {. }
$$

B. $\xi_{0}$ and $\xi_{1}$ are successor ordinals, i.e. $\xi_{0}=\lambda_{0}+1$ and $\xi_{1}=\lambda_{1}+1$ for some $\lambda_{0}$ and $\lambda_{1}$, and moreover

$$
u=\left\{\left(t, \lambda_{1}\right)\right\} \cup\left\{\left(\lambda_{0}, \xi_{1}\right)\right\} \cup y .
$$

In the remaining cases the sequence $s(x)$ was not defined.

C. $\xi_{0}$ and $\xi_{1}$ both equal zero, $t=0$ and $u=y$.

D. $\xi_{0}=0, \xi_{1}$ is a limit ordinal, $t<\xi_{1}$, and furthermore

$$
u=\{(0, t)\} \cup y .
$$

E. $\xi_{0} \neq 0$, at least one of $\xi_{0}$ and $\xi_{1}$ is not a successor ordinal (i.e. it is either zero or a limit ordinal), and moreover $t<\xi_{1}$ or $t<\xi_{0}$ and

$$
u=\{(0, t)\} \cup\left\{\left(\lambda_{0}, \xi_{1}\right)\right\} \cup y \quad \text { or } u=\left\{\left(t, \xi_{1}\right)\right\} \cup y,
$$

resp., according as $\xi_{0}=\lambda_{0}+1$ for some $\lambda_{0}$ and $\xi_{1} \neq 0$ or this is not the case.

In the remaining cases the truth value of $\Sigma x t u$ is "false" by definition.

With the aid of the predicate just defined, the canonical process is easily formalized. Indeed, denote by Fxy that the ordinal $y$ belongs to the $x$ th class of the process. Then depending on the starting class of ordinals $\xi$ satisfying $F 0 \xi$, the other classes can be defined by transfinite induction as follows:

$\left({ }^{4}\right)$ It would cause no conceptual difficulty to consider more general versions of the canonical process such as, e.g., the one having as its set of steps $S^{*}$, defined at the end of $\$ 5$. We nevertheless think that the reader will prefer the presented way, where he can learn the method of formal transcription of the definition on a relatively simple example, and then, if he desires, extend it to more general cases. 


$$
\begin{aligned}
& x \neq 0 \rightarrow:: F x y \cdot \leftrightarrow: \text { ord } y \&: \\
& (\exists \alpha) \sim(\exists u) \Sigma x a u \\
& \&(\forall a)(\forall u)(\Sigma x a u \rightarrow y \in f(\{\xi: \xi \leq y \& F u \xi\})) \\
& \cdot V \cdot(\forall \alpha)(\exists u) \Sigma x \alpha u \\
& \& y \in f(\{\xi: \xi \leq y \&(\exists \alpha)(\exists u)(\Sigma x \alpha u \& F u \xi \\
& \qquad \& \sim(\exists \lambda)(\lambda<\xi \& F u \lambda))\}) .
\end{aligned}
$$

According to this formula, if $x$ is not like an element of $S$ in the sense described above, Fxy obviously holds if and only if $y$ is an ordinal. Indeed, in this case the first component of the implication in the first term of the disjunction on the right-hand side is false. On the other hand, to explain further the above formula, if $x$ is like an element of $S$ the second term of the mentioned disjunction in it corresponds to the cases in which the sequence $s(x)$ was defined, the first one to those in which it was not. Otherwise it is clear how this formula works if we compare it to the one describing the process in $\$ 5$ (see (5.11)).

The notion of dropping out can easily be defined in terms of the predicate $F$. In fact, if drop $x y$ stands for the clause " $y$ drops out at the step $x$ of the canonical process", then we have

$$
\begin{aligned}
\operatorname{drop} x y . \leftrightarrow & . \operatorname{rank} x \in y \& \sim F x y \& \\
& (\forall \alpha)(\forall u)(\alpha \in y \& \Sigma x \alpha u . \rightarrow F u y) .
\end{aligned}
$$

In the terminology of the previous sections the above formula says that $x$ is the first step in the process with rank $x<y$ the class corresponding to which does not contain $y$. It is simpler to express the fact that $y$ drops out at some step. In fact, we have

$$
(\exists x) \operatorname{drop} x y . \leftrightarrow .(\exists x)(\operatorname{rank} x \in y \& \sim F x y) .
$$

To be able to give a full formalization of the above concepts we should also formalize the predicate $\Sigma$ itself. To do this, a task very simple in principle, is rather dull and tedious, and, after all, not needed for our purposes. We mention here briefly only that, in an appropriate formalization, $\Sigma$ is absolute in the following sense:

Lemma 7.1. If $\left\langle A, \epsilon_{A}\right\rangle$ is a natural model for set theory, then

$$
(\forall x)(\forall t)(\forall u)\left(x, t, y \in A \rightarrow . \Sigma x t u \leftrightarrow \Sigma_{A} x t u\right),
$$

$\Sigma_{A}$ denoting the relativization of $\Sigma$ to $A$.

In fact, as is well known, the ordinals are absolute in the above sense; 
therefore the same is true for finite sets of pairs of ordinals. To extend these considerations to the enlarged canonical process defined on the set $S^{*}$ would require dealing $w$ ith infinite sequences; this is, however, only an illusory difficulty, for these sequences contain only a finite number of nonzero members. We note that what we have done cannot be regarded as the rigorous proof of the above "lemma", which, indeed, is not a mathematical assertion in its present form. This can be achieved only after the formalization of $\Sigma$.

Absoluteness of other expressions will also be necessary. In particular, we shall need

Lemma 7.2. The expressions

$$
\begin{gathered}
(\exists \alpha) \sim(\exists u) \Sigma x \alpha u, \\
\left(\forall^{\prime} \alpha\right)(\exists u) \Sigma x \alpha u,
\end{gathered}
$$

featuring in the definition of the predicate $F$, are also absolute in the sense of Lemma 7.1.

We remark here that, in view of the presence of the quantifiers, the absoluteness of these expressions is not a consequence of the similar property of $\Sigma$, but must be derived independently from that. This can be easily done by observing that both classes of those $x$ for which, respectively, the first or the second of the above formulas is true can be described directly, without the quantifiers used. Indeed, the first formula is true if $x$ has one of the forms described in paragraphs $\mathrm{C}, \mathrm{D}$ and $\mathrm{E}$ at the beginning of this section, while the truth-domain of the second formula corresponds to the cases $\mathrm{A}$ and $\mathrm{B}$.

8. Definability of inaccessible cardinals in terms of sets of lower rank. To state in a more precise form the problem mentioned in the title, a problem closely related to our previous considerations, we advance the following

Definition 8.1. The set $c$ is called definable in terms of sets of lower rank if there exists a formula $\phi(x)$ written in finite-length first order logic, containing only $x$ as a free variable and, possibly, constant sets that are stipulated to have smaller ranks than $x$ has, such that all its bound variables are confined to sets of rank less than that of $x$, and which is satisfied if and only if $x=c$.

We add that confining a variable $y$ to sets of rank less than that of $x$ means that the quantifiers corresponding to $y$ occur in their environment in one of the following two ways:

$$
\begin{gathered}
(\forall y)(\operatorname{rank} y<\operatorname{rank} x \rightarrow \ldots), \\
(\exists y)(\operatorname{rank} y<\operatorname{rank} x \& \ldots) .
\end{gathered}
$$


In the above definition we did not specify whether the formula $\phi(x)$ is written in nonsimple or simple first order calculus, i.e. whether or not free predicate variables are allowed to occur in it, respectively.(5) In our cases it will turn out, though, that we can confine ourselves to the simple calculus. That in either case the inaccessible cardinals definable in terms of sets of lower rank form only a very thin collection of the class of all cardinals is confirmed by the following theorem.

Theorem 8.2. If $a$ is an inaccessible cardinal, then those inaccessible cardinals preceding it which are definable in terms of sets of lower ranks form a set nonstationary with respect to $a$.

Indeed, it is easy to verify that the function which assigns to every such cardinal $\xi<\alpha$ the maximum of the ranks of the constants occurring in the formula defining $\xi$ is a regressive function divergent with respect to $\alpha$, in accordance with the assertion of the theorem. It is interesting to compare the above theorem with Theorem 6.2 in the light of our next result, which is a positive assertion on the definability of certain inaccessible cardinals in terms of lower ranks.

Theorem 8.3. If the cardinal a drops out in the canonical process started on the class of all inaccessible cardinals that repeats the fixed-point operation, then a is definable in terms of sets of lower rank.

Using the ideas of the proof below the reader may easily verify the above assertion even for some operations stronger than the fixed-point operation. Such operations can be defined, for example, as follows: For an operation $f$ denote by drop $f$ the operation which discards exactly those elements of a class $H$ which drop out if we perform the canonical process repeating the operation $f$ on $H$ as starting class. Then the fixed-point operation $\mathrm{fp}$, and all the operations drop $\mathrm{f}$, drop drop fp, ..., ad inf are of the desired kind. Another way of generalizing the assertion of the above theorem may be obtained by extending the canonical process if possible. We do not know whether all the inaccessible cardinals definable in terms of sets of lower ranks are obtained by the combination of the two mentioned methods, though we think the answer is negative. The chief obstacle in deciding this problem might be to give a precise definition to the notion of the canonical process.

Proof of the theorem. If $\alpha$ drops out at the step $x_{0}$ of the canonical process, then it is not the limit point of the other ordinals dropping out at this same step;

(5) We use the language of set theory with equality and elementhood. For our purposes, an additional predicate "inacc $x$ " is needed, saying that $x$ is an inaccessible cardinal. 
hence there exists an ordinal $\beta<\alpha$ such that $\alpha$ is the least ordinal dropping out at the step $x_{0}$ and exceeding $\beta$. The formalization of this assertion will be the desired sentence defining the cardinal $\alpha$.

We elaborate the above idea more carefully. To start with, the formula (7.7) defining the process can be rewritten in the mentioned case as follows:

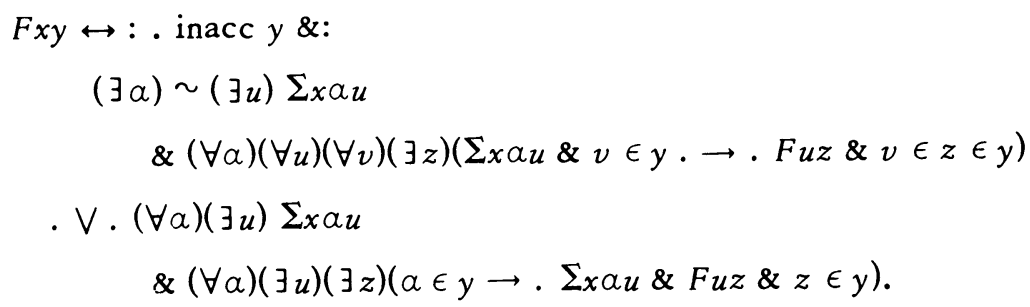

The notation inacc $y$ here expresses that $y$ is an inaccessible cardinal. The above formula can easily be obtained by taking into account the definition of the fixed-point operation. Since the predicate "inacc" was adopted in our language (see footnote (5)), it will be clear that Fxy can be formalized in terms of sets of rank less than the maximum of the rank of $x$ plus one and the rank of $y$.

Therefore it is easy to see that we can formalize the sentence "the ordinal $y$ exceeds $\beta$, and drops out at the step $x_{0}$ " in terms of sets of rank less than $y$. (Note that the above sentence implies that the rank of $x_{0}$ precedes $y$.) Denote the formula corresponding to the above sentence by $\phi(y)$. Then the expression

$$
\phi(y) \& \sim(\exists w)(w \in y \& \phi(w))
$$

defines the ordinal $a$ as the unique set $y$ satisfying it in the desired way, except that it is written in nonsimple first order calculus.

This situation, however, might easily be remedied, since, as is immediately checked, in case rank $x<y$ we have

$$
\begin{aligned}
F x y \leftrightarrow(\forall z) & (\text { rank } z<y \& z<x . \\
& \rightarrow(\forall v)(\exists w)(v \in y \rightarrow . v \in w \in y \& F z w),
\end{aligned}
$$

where we recall that the sign $\prec$ denotes the ordering of the steps of the process; or, with a slight change, if $F(\xi)$ denotes the set of all pairs $\langle z, w\rangle$ such that Fzw holds and the ranks of both $z$ and $w$ precede $\xi$, we can write

$$
F x y \leftrightarrow\left(\nvdash^{\prime} z\right)(\forall v)(\exists w)(\exists \xi)
$$

$$
\text { (rank } z \in y \& z \prec x \& v \in y . \rightarrow . v \in w \in \xi \in y \&\langle z, w\rangle \in F(\xi) \text { ). }
$$

The meaning of this formula does not change if we confine all its quantified variables to sets of rank $<y$. Even the constant $F(\xi)$ has rank $<y$, as easily 
follows from the inaccessibility of $y$; furthermore we can completely eliminate it as a constant by defining it with the aid of a slight modification of the formula (8.3). This shows that it is not necessary to use any new predicate symbol in the formula $\phi$. This observation enables us to define $y$ in the required way with the aid of only the simple first order calculus.

9. Strong incompactness of inaccessible cardinals. Hanf in his cited paper proved that his process of repeating an operation which plays there the role of our stationary-point operation results in a class of strongly incompact cardinals (see [7, Theorem 5, p. 319]). Analogously, here we have the following

Theorem 9.1. All the inaccessible cardinals that drop out in the course of the canonical process started on the class of all inaccessible cardinals and repeating the stationary-point operation are strongly incompact.

This theorem could be established as a corollary of Hanf's if we were able to prove the surmise that our canonical process can be imitated in Hanf's scheme. We can, however, follow a direct way of establishing it, which we think is more illuminating, and can also be generalized (see Theorem 10.2; this theorem still might be a particular case of Hanf's). The idea behind this proof is based on Hanf's, but we hope to present his technique in a more lucid way.

Proof of the theorem. In order to prove the strong incompactness, i.e. to refute the weak compactness of the cardinals concerned we have only to construct for every such cardinal $\alpha_{0}$ an axiom-system consisting of $\alpha_{0}$ axioms written in the language $L_{a_{0}}$ such that the whole is not satisfiable while each of its subsystems of cardinality $<\alpha_{0}$ is so. In order to be able to confine ourselves to natural models (see 2.4) we adopt (2.4) and (2.5) as axioms in our structure

$$
\langle A, \epsilon, \Sigma, F, B\rangle
$$

where the meaning of the predicates listed will be explained gradually.

For a start, $\epsilon$ is a binary predicate playing the role of the relation for elementhood in the model, and for it, apart from the axioms already mentioned, we adopt all the axioms of Zermelo-Fraenkel set theory except the axiom of extensionality, which we have already got as (2.4). The adoption of these axioms will enable us to relativize any set the oretical notion to our model.

The ternary predicate $\Sigma$ will correspond to the relation $\Sigma$ introduced in $\S 7$; the relativized form of its definition given there will be adopted as an axiom. To actually write down this axiom we first had to formalize the definition of $\Sigma$. What is relevant here is only that this can be done.

The binary predicate $F$ will implement the relativized form of the canonical 
process started on the class of all inaccessible cardinals and repeating the stationary-point operation. The axiom defining $F$ can be written on the basis of (7.7) and of the definition of the stationary point operation as follows:

$$
\begin{aligned}
& \text { Fxy } \leftrightarrow: \text {. inacc } y \text { \&: } \\
& (\exists \alpha) \sim(\exists u) \Sigma_{x} \alpha u \\
& \&(\forall a)(\forall u)\left(\Sigma x a u \rightarrow \sim(\exists b) \text { (band } b \& \bigcup_{b}=y \&\right. \\
& (\forall \xi)(\xi \epsilon b \rightarrow \sim F u \xi)) \\
& \text {. V. }(\forall a)(\exists u) \sum x \alpha u \\
& \text { \& }(\forall b) \text { (band } b \& \bigcup_{b}=y \text {. } \\
& \rightarrow(\exists \alpha)(\exists u)(\exists \xi)\left(\Sigma_{x} \alpha u \& F u \quad \xi \& \xi \epsilon b \&\right. \\
& \sim(\exists \lambda)(\lambda \epsilon \xi \& F u \lambda))),
\end{aligned}
$$

where band $b$ means that $b$ is a band (in the model, see 2.12), i.e. is the abbreviation of the right-hand side of

$$
\text { band } b \leftrightarrow(\forall z)\left(z \subseteq b \rightarrow . \bigcup_{z} \epsilon b \vee b \subseteq \bigcup_{z}\right)
$$

We intend to build our model so that the ordinals in it coincide with all the ordinals preceding $\alpha_{0}$, which, we remind the reader, is the ordinal to be proved strongly incompact. As Hanf observed, it is easy to stipulate in the language $L_{a_{0}}$ that any ordinal $\xi<a_{0}$ belongs to our model. Indeed, the formula

$$
\phi_{\xi}\left(x_{\xi}\right)=\left(\exists x_{\eta}\right)_{\eta \leq \xi} \bigwedge_{\mu \leq \xi}(\forall z)\left(z \in x_{\mu} \leftrightarrow \bigvee_{\nu<\mu} z=x_{\nu}\right)
$$

is satisfied in an arbitrary natural model if and only if $x=\xi$. So the axioms

$$
(\exists x) \phi_{\xi}(x),
$$

where $\xi$ runs over all the ordinals $<\alpha_{0}$ will secure that all ordinals $\xi<\alpha_{0}$ are contained in the object-set $A$ of our model. To prevent $\alpha_{0}$ (and so all the ordinals $\geq a_{0}$ ) from being admitted into the model is somewhat more difficult, and needs a device similar to that of defining sets in terms of sets of lower ranks, except that here for the definition of $\alpha_{0}$ there are $\alpha_{0}$ sentences available. Intuitively, we shall represent $\alpha_{0}$ as the supremum of a band $B$ whose existence forces it to drop out at the step $x_{0}$ of the process and require that all the ordinals belonging to the closure of $B$ drop out before $x_{0}$. Since the process in a model can work only slower than in "reality", this will prevent our model from containing $\alpha$. 
More precisely, if the ordinal $\alpha_{0}$ drops out at the step $x_{0}$ then there is a band $B$ with supremum $\alpha_{0}$ all the elements of which have dropped our previously. (If an ordinal is not contained in the starting class of the process, then it is regarded as dropping out in the first step.) The band $B$ can be represented in our model as a unary predicate $B$. To define this predicate we adopt the axioms

$$
(\exists x)\left(\phi_{\xi}(x) \& B x\right) \text { or }(\exists x)\left(\phi_{\xi}(x) \& \sim B x\right),
$$

where $\xi$ runs over all the ordinals $<\alpha_{0}$, according as $\xi \in B$ or $\xi \notin B$ occur. (We note that these axioms actually make those listed under (9.5) superfluous and therefore omittable.) To stipulate that $B$ is a band, or rather that it is a closed set, we add

$$
(\forall x)((\forall y)(y \in x \rightarrow B y) \rightarrow B(\bigcup x))
$$

as an axiom.

To be able to require that all the elements of $B$ drop out before the step $x_{0}$ it is necessary to give a description of $x_{0}$. This, however, can be easily done. In fact, since $x_{0}$ is built up of a finite number of ordinals $<\alpha$ in a "finite" way, it is easy to see that there exists a formula $\phi_{x_{0}}(x)$ which is satisfied if and only if $x=x_{0}$. Thus by the axiom

$$
(\forall \xi)(\exists \lambda)(\exists u)(\exists x)\left(B \xi \rightarrow . \phi_{x_{0}}(x) \& \lambda<\xi \& \Sigma x \lambda u \& \sim F u \xi\right)
$$

we can achieve exactly what we wanted, i.e. that all the elements $>$ rank $x_{0}$ of $B$ drop out before the step $x_{0}$.

We shall now show that the axioms already listed stop our model from containing the ordinal $a_{0}$. In fact, supposing temporarily that $\alpha_{0}$ belongs to the model, it belongs to the closure of the set of ordinals $\xi$ for which $B \xi$ is stipulated under (9.6), and thus by (9.7) we have $B \alpha_{0}$. So, relativizing our process to the model, by axiom (9.8) we see that $\alpha_{0}$ drops out before the step $x_{0}$ in the relativized process; this, however, contradicts the fact that in the nonrelativized process $\alpha_{0}$ drops out at the step $x_{0}$, since the process is only weakened (i.e. made less effectively discarding) by relativization.

To elaborate this least statement more precisely, we shall distinguish between the notation of the relativized and nonrelativized form of the process by writing for the former $F_{A}$. Then by saying that the process is only weakened by relativization we claim that

$$
(\forall x)(\forall y)\left(x, y \in A \& F x y . \rightarrow F_{A} x y\right) .
$$

This can easily be verified, since in the definition (9.2) of the process, apart from the phrase "inacc $y$ ", which is weakened by relativization, and from the absolute predicate $\Sigma$, and from the absolute expressions of Lemma 7.2, and 
apart from the quantifiers that are easily proved to have the same range in the model as in reality, the only critical component occurring is a statement for all bands; now if this statement is true for every band in reality, it is certainly true for every band in the model, proving that our process is in fact weakened by relativization.

So far we know that our model, if it exists, must contain as ordinals exactly those preceding $a_{0}$. Thus, by virtue of the axioms listed under (9.6), the class $B$ has no supremum in the model; hence the axiom

$$
(\exists \xi)(\forall x)(B x \rightarrow x \in \xi)
$$

makes the satisfiability impossible of the axiom system, the construction of which is now accomplished. Nevertheless, if we consider only less than $\alpha_{0}$ axioms of this system, the remaining axioms of the group listed under (9.6) do not determine $B$ for large ordinals $<a_{0}$, therefore such a system can always be satisfied on the set of all sets of rank $<a_{0}$. This proves the strong incompactness of $a_{0}$.

10. Axiomatic investigations relevant to the canonical process. In $\$ 5$ and also later the question cropped up whether the canonical process can be continued still further than it actually was. For the decision of such a problem it is, however, necessary to specify the notion of canonical process. Being unable to do so we do our level best by listing axioms which will partly characterize this notion, but not, as might be expected, up to an isomorphism in the sense given at the end of this section. Nevertheless, by carrying out this program we are able to produce definitely positive achievements, such as generalizations of Theorem 9.1 and of some other minor results. In fact, the most plausible selection principles which spring to mind in deciding which properties of the processes defined in $\$ 5$ are important are a careful analysis of the proof of Theorem 9.1, and a look for those properties of the predicate $\Sigma$ which were indispensable for the validity of the proof.

To start with, we list properties of $\Sigma$ which were not only relevant in this proof, but will also be required in order to be able to define a process with the aid of formula (7.7) which belongs to the family described in $\$ 4$. Listing such properties we first stipulate that

A. $\sum x t u$ implies that $t$ is an ordinal, i.e.

$$
(\forall x)(\forall t)(\forall u)\left(\sum x t u \rightarrow \text { ord } t\right) .
$$

B. If $\Sigma x t u$, then $u$ is uniquely determined by $t$ and $x$, i.e.

$$
(\forall x)(\forall t)(\forall u)(\forall v)\left(\sum x t u \& \sum x t v . \rightarrow u=v\right) .
$$

C. The binary relation $\prec$ defined as the minimal relation satisfying 


$$
(\forall u)(\forall x)((\exists t) \Sigma x t u \rightarrow u \prec x),
$$

can be extended uniquely to a well-ordering of the class $S$ of all sets $u$ such that

$$
(\exists x)(\exists t)\left(\Sigma_{x t u} \vee \Sigma_{u t x}\right) \text {. }
$$

For this extended relation we adopt the same symbol, and, as an aesthetical condition, we add that the initial element of the class $S$ should be the empty set.

This requirement hits two different targets: Firstly, it defines the underlying well-ordering of the class $S$ corresponding to the set $S$ in $\$ 4$; secondly, it gives a meaning to the formula (7.7) as a transfinite-inductive definition.

As an important consequence of the last of the above requirements we may notice that we have

$$
(\forall x)(\forall y)\left(y \prec x \rightarrow(\exists t)(\exists u)\left(\sum x t u \& y \preceq u\right)\right) .
$$

To verify this assume that for some $x$ and $y$ the second component of the above implication is false. This entails that $y \prec x$ cannot be derived from (10.3) with the aid of the transitivity property of the extended relation $\prec$. Therefore, if there is any well-ordered extension of the minimal relation defined by (10.3) there must be one for which $y \prec x$ does not hold. Since this is the unique well-ordered extension of the relation in question, we have shown $\sim y \prec x$.

Our next property does not actually hold for the relation $\Sigma$ as defined in $\S 7$ in case of the canonical process, but the reader may see without any difficulty that the definition given there can be adjusted by changing the relation $\Sigma$ so that this property will be satisfied and also the flow of the process will remain basically unaltered. We did not make this adjustment before because all the important consequences of the next property were verified directly, and therefore it would have caused only additional complications.

D. For the set $x$ and $y$, and for every ordinal $\alpha$ we have

$$
\begin{aligned}
&(\exists \xi) \sim(\exists u)\left(\Sigma_{x \xi u}\right. \rightarrow . \\
&\left.\Sigma x \alpha y \leftrightarrow(\exists z)(\forall \xi)(\exists u)\left(\Sigma z \xi u \& \Sigma_{z \alpha y \&(}(\exists \beta)(\alpha<\beta \& \Sigma z \beta x)\right)\right) .
\end{aligned}
$$

E. $\Sigma$ connects the ordering $\prec w i t h$ the ordering of the ordinals in a compatible way such that

$$
\Sigma x \alpha y \& \Sigma x \beta z \& \alpha<\beta . \rightarrow y<z
$$

holds for every value of the free variables, and furthermore, the class of all $y$ for which

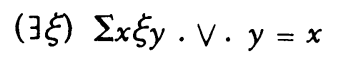


is closed in the ordering of the class $S$ defined under (10.4) for any value of $x$. This, together with the next stated requirement will have very important consequences.

F. The assigned sequences do not mingle in the sense of $\$ 4$, i.e. for any $x$, $y, z$ and $\alpha$ we have

$$
\begin{gathered}
\Sigma x a y \& y \prec z \prec x \&(\forall \xi)(\exists u) \Sigma z \xi u . \\
\rightarrow(\forall \xi)(\forall u)(\Sigma z \xi u \rightarrow y \preceq u) .
\end{gathered}
$$

This nonmingling property and the closedness of the assigned sequences mentioned immediately before it enables us to use the formulae (8.3) and (9.2) to define the process in the particular cases dealt with in $\$ \$ 8$ and 9 , respectively; otherwise we should use the formula (7.7) with appropriate substitutions for the starting class and the repeated operation. Here we dispense with the rather obvious verification of these facts and only mention that they would not necessarily continue to be valid without, for example, the closedness of the assigned sequences. Indeed, in this latter case whether an ordinal was recollected or not would not be determined by the class of all smaller ordinals recollected. This might spoil any regularity property of the classes of ordinals obtained in the course of the process.

In order to be able to define important concepts such as for example that of dropping-out for the present, generalized version of the process we shall introduce the notion of grade, which will stage the steps of the process in a similar way to how it was done with the aid of the ranks of the steps previously. The grade of a step will be defined by putting

$$
\text { grade } x=\sup \{\alpha+1:(\exists y)(\exists u)(u \prec x \prec y \& \Sigma y \alpha u)\} .
$$

At the first sight it might not be clear whether the above definition is always meaningful; indeed, the problem is whether the class of ordinals a satisfying the formula on the right-hand side in the classifier $\left.{ }^{6}\right)$ is limited from above. This is not a priori the case, since the well-ordering $\prec$ is defined on what may be a real class; thus the step $x$ in the above formula may be preceded by a real class of steps. However, a simple consideration invoking condition $F$ decides the question of the correctness of the above definition affirmatively.

The notion of dropping-out can be defined with the aid of the just introduced grading of the steps analogously to (7.8):

(10.11) drop $x y . \leftrightarrow$. grade $x \in y \& \sim F x y \&(\forall a)(\forall u)(\alpha \in y \& \Sigma x \alpha u . \rightarrow F u y)$.

Just as in $\$ 9$ the notion of rank helped in the relativization of the process, so here we can use the grading of steps to truncate the process by omitting

${ }^{(6)}$ By classifier we mean the array of symbols $\{:\}$. 
high-graded steps. More precisely, for any ordinal $\alpha$ define the $\alpha$-confinement $\Sigma_{\alpha}$ of the relation $\Sigma$ such that $\Sigma_{\alpha} x \xi y$ holds if and only if $\Sigma x \xi y$ holds, and

$$
\text { grade } x, \text { grade } y<\alpha \text {. }
$$

(This in particular implies also $\xi<\alpha$.) The $\alpha$-truncation of the process will be be defined by the formula (7.7), if we replace all occurrences of $\Sigma$ by $\Sigma_{\alpha}$ there, and confine all ordinal variables to ordinals $<a$.

It is easy to establish that the $\alpha$-truncation of the process has the same effect on ordinals $<\alpha$ as the whole process has. The critical point here is to observe that the recollection steps do not move by truncation, i.e. that if grad $x$ $<\alpha$, then

$$
(\forall \xi)(\exists u) \Sigma x \xi u \leftrightarrow(\forall \xi)(\exists u)\left(\xi<a \rightarrow \Sigma_{\alpha} x \xi u\right)
$$

This, however, easily follows from the definition of grading by recalling condition D.

The above conditions will enable us to generalize Theorem 9.1. In the direction of showing this the first step is to verify

Lemma 10.1. For any regular cardinal number a the $\alpha$-truncation of a process defined with the aid of a relation $\Sigma$ satisfying the above conditions A-F contains at most a steps.

In other words, the number of steps of grade $<\alpha$ is at most $\alpha$. It is interesting to compare the proof of this lemma with that of Theorem 4.1.

Proof. Assume the contrary of the assertion of the lemma, i.e. assume that for some regular ordinal $\alpha$ the $\alpha$-truncation of a process satisfying the above axioms $A-F$ has more than a steps, and omit the too faraway steps so that the order type of the set of the remaining steps in the ordering $\prec$ be exactly $\alpha^{+}, \alpha^{+}$ as before denoting the cardinal immediately following $\alpha$. Then, as is easily seen, the restriction $\Sigma^{\prime}$ of the relation $\Sigma$ to the retained steps will satisfy the same axioms A-F (except D) if we confine all the ordinal variables there to ordinals preceding $\alpha$.

Now every element cofinal to $\alpha$ in the ordering $\prec$ of the set of the retained steps must be a recollection step. Indeed, with the aid of the relativized form of (10.5) we can derive

$$
(\forall \xi)(\exists u)(\xi<\alpha \rightarrow \Sigma x \xi u)
$$

for every such element $x$. Therefore, using the nonmingling property, it is easily verified that the function which assigns to every such element $x$ the element $y$ with $\Sigma x 1 y$ is one-to-one, and obviously regressive. These two facts together imply that this function is also divergent with respect to $\alpha^{+}$, i.e. 
its domain is nonstationary with respect to $\alpha^{+}$. This is, however, wrong (see footnote $\left({ }^{2}\right)$ on $\left.\mathrm{p} .78\right)$ proving the assertion of the lemma.

We are now in position to state the promised generalization of Theorem 9.1.

Theorem 10.2. All the inaccessible cardinals that drop out in the course of a process defined with the aid of a ternary relation $\Sigma$ satisfying the axioms A-F, started on the class of all inaccessible cardinals, and repeating the stationary. point operation, are strongly incompact.

The proof is almost the same as it was in the special case. The only deviation from the line of the proof given there is at the definition of the predicate $\Sigma$; this is the predicate to implement the $\alpha$-truncation of the process, where $\alpha$ is the cardinal being proved to be strongly incompact. Since, as proved above, the $\alpha$ truncation of the process contains at most $\alpha$ steps, by changing the process within isomorphism we can select these steps from among ordinals $<\alpha$. Thus $\Sigma$ will be a relation over ordinals $\langle\alpha$ and for every triple $\langle\xi, \lambda, \mu\rangle$ of such ordinals it can be told by a separate axiom whether $\Sigma \xi \lambda \mu$ or $\sim \Sigma \xi \lambda \mu$ holds. Such an axiom will use the technique of defining ordinals $<\alpha$ uniquely in the language $L_{\alpha}$ as elaborated in the previous section. Apart from the se axioms the axioms $\mathrm{A}-\mathrm{F}$ also ought to be included in the axiom system used in the proof of the theorem in order to be able to ward off the entering of alien steps represented by ordinals $\geq \alpha$ into our model, which must be considered as a possibility until we prove that no ordinal $\geq \alpha$ can belong to the model.

There are other theorems, e.g. Theorem 6.2 and its corollary which can also be generalized for all processes satisfying the axioms A-F. The prospects of the generalization of The orem 8.2 concerning the definability of inaccessible cardinals in terms of sets of lower ranks are, however, much dimmer. Indeed, here it is essential that the $\alpha$-trunction of the process could be defined with the aid of less than a axioms, possibly written in the language $L_{\alpha}$. Therefore, to reach a result in this direction would require looking for axiom-systems which assured unique representability of the relation $\Sigma$ in a certain sense. For example the following property could be considered: Any relation $\Sigma$ satisfying the axiom system in question is isomorphic to a confinement of a particular one of such relations. As yet we do not even know whether our axiom system A-F already satisfies such a property.

\section{REFERENCES}

1. P. J. Cohen, Set theory and the continuum hypothesis, Benjamin, New York, 1966. MR 38 \#999.

2. G. Fodor, Eine Bemerkung zur Theorie der regressiven Funktionen, Acta Sci. Math. (Szeged) 17 (1956), 139-142. MR 18, 551. 
3. G. Fodor, On a process concerning inaccessible cardinals. I, Acta Sci. Math. (Szeged) 27 (1966), 111-124. MR 34 \#67.

4. - On a process concerning inaccessible cardinals. II, Acta Sci. Math. (Szeged) 27 (1966), 129-140. MR 35 \#2743.

5. - On a process concerning inaccessible cardinals. III, Acta Sci. Math. (Szeged) 28 (1967), 197-200. MR 36 \#54.

6. H. Gaifman, A generalization of Mahlo's method for obtaining large cardinal numbers, Israel J. Math. 5 (1967), 188-200. MR 36 \#4999.

7. W. Hanf, Incompactness in languages with infinitely long expressions, Fund. Math. 53 (1963/64), 309-324. MR 28 \#3943.

8. H. J. Keisler and A. Tarski, From accessible to inaccessible cardinals: Results holding for all accessible cardinal numbers and the problem of their extension to inaccessible ones, Fund. Math. 53 (1963/64), 225-308. MR 29 \#3385.

9. A. Lévy, Axiom schemata of strong infinity in axiomatic set theory, Pacific J. Math. 10 (1960), 223-238. MR 23 \#A1522.

10. P. Mahlo, Über lineare transfinite Mengen, Ber. Verh. Königl. Sächs. Ges. Wiss. Leipzig Math.-Phys. K1. 63 (1911), 187-225.

11. — Zur Theorie und Anwendungen der $\delta$-Zahlen, Ber. Verh. Königl. Sächs. Ges. Wiss. Leipzig Math.-Phys. K1. 64 (1912), 108-112.

12. —_, Zur Theorie und Anwendungen der $\delta$-Zahlen, Ber. Verh. Königl. Sächs.

Ges. Wiss. Leipzig Math.-Phys. K1. 65 (1913), 268-282.

13. A. Mostowski, An undecidable arithmetical statement, Fund. Math. 36 (1949), 143-164. MR 12, 2.

14. J. von Neumann, Zur Einführung der transfiniten Zahlen, Acta Sci. Math. (Szeged) $1(1922 / 23), 199-208$.

15. W. Neumer, Verallgemeinerung eines Satzes von Alexandroff und Urysohn, Math. Z. 54 (1951), 254-261. MR 13, 331.

16. J. C. Shepherdson, Inner models for set theory. I, J. Symbolic Logic 16 (1951), 161-190. MR 13, 522.

17. A. Tarski, Remarks on predicate logics with infinitely long expressions, Colloq. Math. 6 (1958), 171-176. MR 20 \#6351.

18. A. Tarski, Some problems and results relevant to the foundations of set theory, Logic, Methodology and Philosophy of Science (Proc. 1960 Internat. Congr.), Stanford Univ. Press, Stanford, Calif., 1962, pp. 125-135. MR 27 \#1382. 